\title{
A GRADIENT-BASED APPROACH TO A CLASS OF HYBRID OPTIMAL CONTROL PROBLEMS
}

\author{
Vadim Azhmyakov * Jörg Raisch ${ }^{*, * *}$ \\ ${ }^{*}$ Fachgebiet Regelungssysteme, Institut für Energie- und \\ Automatisierungstechnik, Technische Universität Berlin, \\ Einsteinufer 17, D-10587 Berlin, Germany. \\ e-mail: azhmyakov@control.tu-berlin.de \\ ** Systems and Control Theory Group, Max Planck Institute for \\ Dynamics of Complex Technical Systems, Sandtorstr. 1, \\ D-39106 Magdeburg, Germany. \\ email: raisch@control.tu-berlin.de and \\ raisch@mpi-magdeburg.mpg.de
}

\begin{abstract}
We investigate optimal control problems for a class of non-stationary hybrid systems with autonomous location transitions. Using the Lagrange approach and the technique of the reduced gradient, we derive necessary optimality conditions for the considered class of problems. These optimality conditions are closely related to a variant of the Hybrid Maximum Principle and can be used for constructive optimization algorithms. Copyright $(2006$ IFAC
\end{abstract}

Keywords: hybrid systems, optimal control, computational algorithms.

\section{INTRODUCTION}

The optimal control of hybrid systems has become a new focus of nonlinear control theory (see e.g., $[4,5,6,12,13,17,18])$. Hybrid control systems are mathematical modes of heterogeneous systems consisting of a continuous part, a finite number of continuous controllers and a discrete supervisor. For a hybrid optimal control problem, the main tool toward the construction of optimal trajectories is the Hybrid Maximum Principle [6,12,13,17,18]. This result generalizes the classical Pontryagin Maximum Principle $[3,10,15]$. It is well-known that the standard proof of the Pontryagin Maximum Principle is based on the techniques of "needle variations" (see e.g., $[10,15])$. The character of a general hybrid optimal control problem changes the possibility of using the standard needle variations [13]. Therefore, a variant of the Hybrid Maximum Principle for a hybrid optimal control problem can be proved only under some restrictive assumptions (see e.g., $[12,13,17,18]$ ). In effect, these assumptions guarantee that the classical needle variations are still admissible variations. Furthermore, in the context of a practical implementation of the Hybrid Maximum Principle we need to construct a simultaneous solution of a large-dimensional boundaryvalue problem and of a family of sophisticated auxiliary minimization problems. This is a complicated problem, especially in the case of many-dimensional systems with a lot of switchings.

In this paper, we consider a class of non-stationary hybrid control systems with autonomous (uncontrolled) location transitions. For general theory of hybrid systems and basic definitions we refer to, e.g., $[2,11,16]$. Using an approach based on Lagrange-type techniques and on reduced gradients, we obtain a set of first-order necessary optimality conditions for the above class of nonlinear hybrid optimal control problems. The explicit computation of the corresponding reduced gradients provides also a basis for applications of some ef- 
fective gradient-based optimization algorithms to the given hybrid optimal control problems.

The remainder of the paper is organized as follows. Section 2 contains the initial hybrid optimal control problem and some basic facts. Section 3 is devoted to the concepts of reduced gradients for optimal control problems in abstract and specific hybrid settings. In Section 4 we present the necessary optimality conditions and propose a gradient-based computational approach to the initial problem. Section 5 summarizes the article.

\section{PROBLEM FORMULATION}

We start by introducing a variant of the standard definition of hybrid systems $[12,13,17,18]$.

Definition 1. A hybrid system is a 7-tuple

$$
\{Q, M, U, F, \mathcal{U}, I, \mathcal{S}\},
$$

where

- $Q$ is a finite set of discrete states (called locations);

- $M=\left\{M_{q}\right\}_{q \in Q}$ is a family of smooth manifolds, indexed by $Q$;

- $U \subseteq \mathbb{R}^{m}$ is a set of admissible control input values (called control set);

- $F=\left\{f_{q}\right\}, q \in Q$ is a family of maps

$$
f_{q}:[0,1] \times M_{q} \times U \rightarrow T M_{q},
$$

where $T M_{q}$ is the tangent bundle of $M_{q}$ (see e.g., $[7,10])$

- $\mathcal{U}$ is the set of all admissible control functions;

- $I=\left\{I_{q}\right\}$ is a family of subintervals of $[0,1]$ such that the length of each $I_{q}$ is less than 1;

- $\mathcal{S}$ is a subset of $\Xi$, where

$$
\Xi:=\left\{\left(q, x, q^{\prime}, x^{\prime}\right): q, q^{\prime} \in Q, x \in M_{q}, x^{\prime} \in M_{q^{\prime}}\right\}
$$

A hybrid system from Definition 1 is defined on the time-interval $[0,1]$. Note that in contrast to the general definition of a hybrid system $[12,13,17,18]$, the control set $U$ from Definition 1 is the same for all locations. Moreover, in the sense of this definition the set $\mathcal{U}$ is also independent of a location. Let us assume that $U$ is a compact set and

$$
\mathcal{U}:=\left\{u(\cdot) \in \mathbb{L}_{m}^{2}([0,1]): u(t) \in U \text { a.e. on }[0,1]\right\},
$$

where $\mathbb{L}_{m}^{2}([0,1])$ is the standard Lebesgue space of all square-integrable functions $u:[0,1] \rightarrow \mathbb{R}^{m}$. We now introduce some additional hypothesis for the vector fields $f_{q}, q \in Q$ :

- all functions $f_{q}(t, \cdot, \cdot)$ from $F$ are differentiable,

- $f_{q}, \partial f_{q} / \partial x, \partial f_{q} / \partial u$ are continuous and there exist constants $C_{q}<\infty$ such that

$$
\begin{aligned}
& \left\|\frac{\partial}{\partial x} f_{q}(t, x, u)\right\| \leq C_{q}, \\
& q \in Q,(t, x, u) \in[0,1] \times M_{q} \times U .
\end{aligned}
$$

For $q, q^{\prime} \in Q$ one can also define the switching set

$$
S_{q, q^{\prime}}:=\left\{\left(x, x^{\prime}\right) \in M_{q} \times M_{q^{\prime}}:\left(q, x, q^{\prime} x^{\prime}\right) \in \mathcal{S}\right\} .
$$

from location $q$ to location $q^{\prime}$. The intervals $I_{q}, q \in Q$ indicate the lengths of time intervals on which the system can stay in location $q$. We say that a location switching from $q$ to $q^{\prime}$ occurs at a switching time $t^{\text {switch }} \in[0,1]$. We now consider a hybrid system with $r \in \mathbb{N}$ switching times $\left\{t_{i}\right\}, i=1, \ldots, r$, where

$$
0=t_{0}<t_{1}<\ldots<t_{r}<t_{r+1}=1 .
$$

Note that the sequence of switching times $\left\{t_{i}\right\}$ is not defined a priory. A hybrid control system remains in location $q_{i} \in Q$ for all $t \in\left[t_{i-1}, t_{i}[, i=1, \ldots, r+1\right.$. Let $t_{i}-t_{i-1} \in I_{q_{i}}$ for all $i=1, \ldots, r+1$. A hybrid system (in the sense of Definition 1) that satisfies the above assumptions is denoted by $\mathcal{H} S$.

Definition 2. Let $u(\cdot) \in \mathcal{U}$ be an admissible control for a hybrid control system $\mathcal{H} S$. Then a continuous trajectory of $\mathcal{H} S$ is an absolutely continuous function

$$
x:[0,1] \rightarrow \bigcup_{q \in Q} M_{q}
$$

such that $x(0)=x_{0} \in M_{q_{1}}$ and

- $\dot{x}(t)=f_{q_{i}}(t, x(t), u(t))$ for almost all $t \in\left[t_{i-1}, t_{i}\right]$ and all $i=1, \ldots, r+1$;

- the switching condition $\left(x\left(t_{i}\right), x\left(t_{i+1}\right)\right) \in S_{q_{i}, q_{i+1}}$ holds if $i=1, \ldots, r$.

The vector $\mathcal{R}:=\left(q_{1}, \ldots q_{r+1}\right)^{T}$ is a discrete trajectory of the hybrid control system $\mathcal{H} S$.

Definition 2 describe the dynamic of a hybrid control system $\mathcal{H} S$. Since $x(\cdot)$ is an absolutely continuous function, Definition 2 describe a class of hybrid systems without impulse components of the continuous trajectories. Therefore, the corresponding switching sets $S_{q, q^{\prime}}$ (and $S_{q_{i}, q_{i+1}}$ ) are defined for $x=x^{\prime}$ (for $\left.x\left(t_{i}\right)=x\left(t_{i+1}\right)\right)$.

Under the above assumptions for the given family of vector fields $F$, for each admissible control $u(\cdot) \in$ $\mathcal{U}$ and for every interval $\left[t_{i-1}, t_{i}\right]$ (for every location $\left.q_{i} \in \mathcal{R}\right)$ there exists a unique absolutely continuous solution of the corresponding differential equation. This means that for each $u(\cdot) \in \mathcal{U}$ we have a unique absolute continuous trajectory of $\mathcal{H} S$. Moreover, the switching times $\left\{t_{i}\right\}$ and the discrete trajectory $\mathcal{R}$ for a hybrid control system $\mathcal{H} S$ are also uniquely defined. Therefore, it is reasonable to introduce the following concept.

Definition 3. Let $\mathcal{H} S$ be a hybrid control system as defined above. For an admissible control $u(\cdot) \in \mathcal{U}$, the triplet $\mathcal{X}^{u}:=(\tau, x(\cdot), \mathcal{R})$, where $\tau$ is the set of the corresponding switching times $\left\{t_{i}\right\}, x(\cdot)$ and $\mathcal{R}$ are the corresponding continuous and discrete trajectories, is called hybrid trajectory of $\mathcal{H} S$. 
Let $\phi: \mathbb{R}^{n} \rightarrow \mathbb{R}$ be a continuously differentiable function. Given a hybrid control system $\mathcal{H} S$ we denote the following Mayer-type hybrid optimal control problem by $O C P$ :

$$
\begin{aligned}
& \operatorname{minimize} \phi(x(1)) \\
& \text { subject to } \dot{x}(t)=f_{q_{i}}(t, x(t), u(t)) \text { a.e. on }\left[t_{i-1}, t_{i}\right] \\
& i=1, \ldots, r+1, x(0)=x_{0} \in M_{q_{1}}, u(\cdot) \in \mathcal{U} .
\end{aligned}
$$

Evidently, (1) is the problem of minimizing the Mayer cost functional $J(\mathcal{X}):=\phi(x(1))$ over all hybrid trajectories $\mathcal{X}$ of $\mathcal{H} S$. Note that we study $O C P$ (1) in the absence of target (endpoint) and state constraints. For necessary optimality conditions for (1) in the form of a Hybrid Maximum Principle we refer to $[5,17]$.

\section{REPRESENTATION OF FUNCTIONAL DERIVATIVES}

\subsection{The General Gradient Formula}

Let us first examine an abstract optimal control problem (the generalization of (1)) which involves a control variable $v$ along with a state variable $\xi$

$$
\begin{aligned}
& \operatorname{minimize} T(\xi, v) \\
& \text { subject to } P(\xi, v)=0, \\
& (\xi, v) \in \Omega,
\end{aligned}
$$

where $T: X \times Y \rightarrow R$ is a cost functional, $X, Y$ are real Banach and Hilbert spaces and

$$
P: X \times Y \rightarrow X
$$

is a given mapping. By $\Omega$ we denote here a nonempty subset of $X \times Y$.

Definition 4. We say that an admissible pair

$$
(\hat{\xi}, \hat{v}) \in \tilde{\Omega}:=\{(\xi, v) \in Q \mid P(\xi, v)=0\}
$$

is a local solution of (2) if

$$
T(\hat{\xi}, \hat{v}) \leq T(\xi, v) \quad \forall(\xi, v) \in W_{(\hat{\xi}, \hat{v})} \subset \Omega,
$$

where $W_{(\hat{\xi}, \hat{v})} \subset X \times Y$ is a neighborhood of $(\hat{\xi}, \hat{v})$.

All derivatives considered in this papers are Fréchet derivatives. We assume that the mappings $T$ and $P$ are continuously differentiable and that the state equation $P(\xi, v)=0$ can be solved with respect to $\xi$, i.e.

$$
\xi=\omega(v),
$$

where $\omega: Y \rightarrow X$ is a differentiable function. In this case the functional $T(\xi, v)$ can be represented as a functional depending only on $v$, namely,

$$
T(\xi, v)=T(\omega(v), v)=\tilde{T}(v) .
$$

Note that the introduced abstract optimal control problem (2) is of primary importance in many applications. Not only the hybrid optimal control problem (1) but also an ODE- and a PDE-optimal control problem or an optimal control problem with integral equations can also be formulated (in various ways) as an abstract problem (2). Moreover, a usual finite-dimensional approximation of an infinite-dimensional $O C P$ has the form of the minimization problem (2). In the above cases the condition $P(\xi, v)=0$ represents the corresponding "state equation" of a specific optimal control problem. Assume that the abstract problem (2) is regular (see [10]). Define the Lagrangian of problem (2)

$$
\mathcal{L}(\xi, v, p):=T(\xi, v)+\langle p, P(\xi, v)\rangle_{X},
$$

where $p \in X^{*}$ and $\langle p,\rangle_{X}: X \rightarrow \mathbb{R}$. Here $X^{*}$ is the (topological) dual space to $X$. For the generalized Lagrange Multiplier Rule see e.g., [10,1]. We use the standard notation

$$
T_{\xi}, T_{v}, P_{\xi}, P_{v}, \mathcal{L}_{\xi}, \mathcal{L}_{p}, \mathcal{L}_{u}
$$

for the partial derivatives of the functions $T, P$ and $\mathcal{L}$. Moreover, we introduce the adjoint operators

$$
T_{\xi}^{*}, T_{v}^{*}, P_{\xi}^{*}, P_{v}^{*}, \mathcal{L}_{\xi}^{*}, \mathcal{L}_{p}^{*}, \mathcal{L}_{u}^{*}
$$

to the corresponding derivatives (linear operators) and also consider the adjoint operator $\nabla \tilde{T}^{*}(v)$ to $\nabla \tilde{T}(v)$. In the context of (2) we now formulate an immediate consequence of the above solvability assumption for the state equation $P(\xi, v)=0$. Note that a usual solvability criterion for this follows from an appropriate variant of the Implicit Function Theorem [7,1].

Theorem 5. Let $T$ and $P$ be continuously Fréchet differentiable and let the state equation in (2) be solvable. Assume that there exists the inverse operator

$$
\left(P_{\xi}^{*}\right)^{-1} \in L\left(\left(X^{*} \times Y^{*}\right), X^{*}\right)
$$

to $P_{\xi}$. Then the gradient $\nabla \tilde{T}^{*}(v)$ can be found by solving the following equations

$$
\begin{aligned}
& P(\xi, v)=\mathcal{L}_{p}^{*}(\xi, v, p)=0, \\
& T_{\xi}^{*}(\xi, v)+P_{\xi}^{*}(\xi, v) p=\mathcal{L}_{\xi}^{*}(\xi, v, p)=0, \\
& \nabla \tilde{T}^{*}(v)=T_{v}^{*}(\xi, v)+P_{v}^{*}(\xi, v) p=\mathcal{L}_{v}^{*}(\xi, v, p) .
\end{aligned}
$$

Let us sketch the proof of Theorem 5. Differentiating the functional $\tilde{T}$ and state equation in (2) we obtain

$$
\begin{aligned}
& P_{\xi}(\xi, v) \nabla \omega(v)+P_{v}(\xi, v)=0, \\
& \nabla \tilde{T}(v)=T_{v}(\xi, v)+T_{\xi}(\xi, v) \nabla \omega(v) .
\end{aligned}
$$

The existence of $\left(P_{\xi}^{*}\right)^{-1}$ implies the formula

$$
\nabla \omega(v)=-\left(P_{\xi}\right)^{-1}(\xi, v) P_{v}(\xi, v) .
$$

Hence

$$
\nabla \tilde{T}(v)=T_{v}(\xi, v)-T_{\xi}(\xi, v)\left(P_{\xi}\right)^{-1}(\xi, v) P_{v}(\xi, v),
$$

and

$$
\nabla \tilde{T}^{*}(v)=T_{v}^{*}(\xi, v)-P_{v}^{*}(\xi, v)\left(P_{\xi}^{*}\right)^{-1}(\xi, v) T_{\xi}^{*}(\xi, v) .
$$

On the other hand, we can calculate $p$ from the second (adjoint) equation in (3) and substitute it to the third (gradient) equation. In this manner we also obtain the given relation (4). Note that a related result was also obtained in [19] for classical optimal control problems. 
3.2 Reduced Gradients for Hybrid Optimal Control Problems

Consider a hybrid control system $\mathcal{H} S$. For an admissible control function $u(\cdot) \in \mathcal{U}$ we obtain the corresponding hybrid trajectory $\mathcal{X}^{u}$. For every interval $\left[t_{i-1}, t_{i}\right]$ from $\tau$ we can define the characteristic function of $\left[t_{i-1}, t_{i}\right]$

$$
\beta_{\left[t_{i-1}, t_{i}\right)}(t)= \begin{cases}1 & \text { if } t \in\left[t_{i-1}, t_{i}\right) \\ 0 & \text { otherwise. }\end{cases}
$$

Using the introduced characteristic functions, we rewrite the state differential equations from Definition 2 for the continuous trajectory $x(\cdot)$ in the following form

$$
\dot{x}(t)=\sum_{i=1}^{r+1} \beta_{\left[t_{i-1}, t_{i}\right)}(t) f_{q_{i}}(t, x(t), u(t)),
$$

where $x(0)=x_{0}$. Under the above assumptions for the family of vector fields $F$, the right-hand side of the obtained differential equation (5) satisfies the conditions of the extended Caratheodory Theorem (see e.g., [9]). Therefore, there exists a unique (absolutely continuous) solution of (5). We now apply the abstract Theorem 5 to the hybrid $O C P$ (1). In the case of the hybrid control system $\mathcal{H} S$ we have

$$
X=\mathbb{W}_{n}^{1, \infty}([0,1]), Y=\mathbb{L}_{m}^{2}([0,1]) .
$$

$\operatorname{By} \mathbb{W}_{n}^{1, \infty}([0,1])$ we denote here the Sobolev space of all absolutely continuous functions with essentially bounded derivatives. Let us introduce the operator

$$
P: \mathbb{W}_{n}^{1, \infty}([0,1]) \times \mathbb{L}_{m}^{2}([0,1]) \rightarrow \mathbb{W}_{n}^{1, \infty}([0,1]) \times \mathbb{R}^{n},
$$

where

$$
\left.P(x(\cdot), u(\cdot))\right|_{t}:=\left(\begin{array}{c}
\dot{x}(t)-\sum_{i=1}^{r+1} \beta_{\left[t_{i-1}, t_{i}\right)}(t) f_{q_{i}}(t, x(t), u(t)) \\
x(0)-x_{0}
\end{array}\right) .
$$

Evidently, the operator equation $P(x(\cdot), u(\cdot))=0$ is consistent with the state equation from the abstract optimization problem (2). Consider a regular $O C P$ (1) and introduce the Hamiltonian

$$
H(t, x, u, p)=\left\langle p, \sum_{i=1}^{r+1} \beta_{\left[t_{i-1}, t_{i}\right)}(t) f_{q_{i}}(t, x, u)\right\rangle .
$$

where $p \in \mathbb{R}^{n}$. Since every admissible control $u(\cdot)$ determines a unique hybrid trajectory $\mathcal{X}^{u}$, the following cost functional $\tilde{J}: \mathcal{U} \rightarrow \mathbb{R}$ such that $\tilde{J}(u(\cdot)):=J\left(\mathcal{X}^{u}\right)$ is well-defined. The differentiability of the given function $\phi$ implies the differentiability of $\tilde{J}$. The corresponding derivative is denoted by $\nabla \tilde{J}$. In the particular case of $O C P$ (1) the evaluation of the adjoint operator $\nabla \tilde{J}^{*}$ to $\nabla \tilde{J}$ is relatively easy. We now present our main result that follows from Theorem 5 .

Theorem 6. Consider a regular OCP (1). The gradient $\nabla \tilde{J}^{*}(u(\cdot))$ can be found by solving the equations

$$
\begin{aligned}
& \dot{x}(t)=H_{p}(t, x(t), u(t), p(t)), x(0)=x_{0}, \\
& \dot{p}(t)=-H_{x}(t, x(t), u(t), p(t)), p(1)=-\phi_{x}(x(1)), \\
& \nabla \tilde{J}^{*}(u(\cdot))(t)=-H_{u}(t, x(t), u(t), p(t)),
\end{aligned}
$$

where $p(\cdot)$ is an absolutely continuous function (an "adjoint variable").

Proof. The Lagrangian of the regular problem (1) can be written as

$$
\begin{aligned}
& \mathcal{L}(x(\cdot), u(\cdot), \hat{p}, p(\cdot))=\phi(x(1))+\left\langle\hat{p}, x(0)-x_{0}\right\rangle+ \\
& +\left\langle p(t), \dot{x}(t)-\sum_{i=1}^{r+1} \beta_{\left[t_{i-1}, t_{i}\right)}(t) f_{q_{i}}(t, x(t), u(t))\right\rangle d t
\end{aligned}
$$

where the adjoint variable here contains two components $\hat{p} \in \mathbb{R}^{n}$ and $p(\cdot)$. If we differentiate the Lagrange function with respect to the adjoint variable, then we obtain the first equation from (6)

$$
\begin{aligned}
& \dot{x}(t)=\sum_{i=1}^{r+1} \beta_{\left[t_{i-1}, t_{i}\right)}(t) f_{q_{i}}(t, x(t), u(t))= \\
& =H_{p}(t, x(t), u(t), p(t)),
\end{aligned}
$$

with $x(0)=x_{0}$. Consider the term

$$
\int_{0}^{1}\langle p(t), \dot{x}(t)\rangle d t
$$

From the integration by part we have

$$
\begin{aligned}
& \int_{0}^{1}\langle p(t), \dot{x}(t)\rangle d t=\langle p(1), x(1)\rangle-\langle p(0), x(0)\rangle- \\
& -\int_{0}^{1}\langle\dot{p}(t), x(t)\rangle .
\end{aligned}
$$

Hence

$$
\begin{aligned}
& \mathcal{L}(x(\cdot), u(\cdot), \hat{p}, p(\cdot))=\phi(x(1))+\langle p(1), x(1)\rangle+ \\
& +\langle\hat{p}-p(0), x(0)\rangle-\left\langle\hat{p}, x_{0}\right\rangle-\int_{0}^{1}\langle\dot{p}(t), x(t)\rangle d t+ \\
& +\int_{0}^{1}\left\langle p(t), \sum_{i=1}^{r+1} \beta_{\left[t_{i-1}, t_{i}\right)}(t) f_{q_{i}}(t, x(t), u(t))\right\rangle d t .
\end{aligned}
$$

If we differentiate $\mathcal{L}$ in (7) with respect to $x(\cdot)$, we can use Theorem 5 and compute $\mathcal{L}_{x}, \mathcal{L}_{x}^{*}$. Thus we obtain the second relation in (6). Using (7), we also write

$$
\mathcal{L}_{u}(x(\cdot), u(\cdot), \hat{p}, p(\cdot)) v(\cdot)=-\int_{0}^{1} H_{u}(t, x(t), u(t)) v(t) d t
$$

for every $v(\cdot) \in \mathbb{L}_{m}^{2}([0,1])$. By Theorem 5 , we obtain the last relation in (6)

$\nabla \tilde{J}^{*}(u(\cdot))(t)=\mathcal{L}_{u}^{*}(x(\cdot), u(\cdot), \hat{p}, p(\cdot))=-H_{u}(t, x(t), u(t))$.

The proof is finished.

The formulated result allows the explicit computation of the gradient $\nabla \tilde{J}$ (or $\left.\nabla \tilde{J}^{*}\right)$ in a sophisticated minimization problem (1).

\section{NECESSARY OPTIMALITY CONDITIONS AND THE COMPUTATIONAL ASPECT}

In Section 3 we have developed explicit formulae for the reduced gradient of the cost functional in (1). To make a step forward in the study of the given $O C P$ we will discuss the necessary optimality conditions for (1) and some related numerical aspects. Let us formulate an easy consequence of Theorem 6. 
Theorem 7. Assume that $O C P(1)$ has an optimal solution $\left(u^{o p t}(\cdot), x^{o p t}(\cdot)\right)$ such that $u^{o p t}(t) \in \operatorname{int}\{U\}$, where $\operatorname{int}\{U\}$ is the interior of the set $U$. Then $\left(u^{o p t}(\cdot), x^{o p t}(\cdot)\right)$ can be found by solving the following equations

$$
\begin{aligned}
& \dot{x}^{o p t}(t)=H_{p}\left(t, x^{o p t}(t), u^{o p t}(t), p(t)\right), \\
& x(0)=x_{0}, \\
& \dot{p}(t)=-H_{x}\left(t, x^{o p t}(t), u^{o p t}(t), p(t)\right), \\
& p(1)=-\phi_{x}\left(x^{o p t}(1)\right), \\
& H_{u}\left(t, x^{o p t}(t), u^{o p t}(t), p(t)\right)=0 .
\end{aligned}
$$

Clearly, the conditions (8) from Theorem 7 present a necessary optimality conditions for a special case of problem (1). Note that the last equation in (8) is consistent with the usual optimality condition $\nabla \tilde{J}^{*}(u(\cdot))(t)=0, t \in[0,1]$ if the optimal control takes values in an open bounded control set.

Alternatively, Theorem 6 and Theorem 7 provide a basis for a wide class of the gradient-based optimization algorithms for (1). We now assume that the control set $U$ has a so-called box-form, namely,

$$
U:=\left\{u \in \mathbb{R}^{m}: b_{-}^{j} \leq u_{j} \leq b_{+}^{j}, j=1, \ldots, m\right\},
$$

where $b_{-}^{j}, b_{+}^{j}, j=1, \ldots, m$ are constants. Let us consider, for example, the standard gradient algorithm in $\mathbb{L}_{m}^{2}([0,1])$ (see e.g., $\left.[8,14]\right)$

$$
\begin{aligned}
& u^{k+1}(t)=u^{k}(t)-\gamma_{k} \nabla \tilde{J}^{*}\left(u^{k}(\cdot)\right)(t), t \in[0,1] \\
& b_{-}^{j} \leq u_{j}^{k+1}(t) \leq b_{+}^{j}, j=1, \ldots, m, k=0,1, \ldots \\
& u^{0}(\cdot) \in \mathcal{U},
\end{aligned}
$$

where $\gamma_{k}$ is a step-size of the gradient algorithm and $\left\{u^{k}(\cdot)\right\} \subset \mathbb{L}_{m}^{2}([0,1])$ is the sequence of iterations. Note that in general cases an admissible iterative control $u^{k+1}(\cdot)$ can also be obtained by a projection

$$
u^{k+1}(t)=P_{U}\left(u^{k}(t)-\gamma_{k} \nabla \tilde{J}^{*}\left(u^{k}(\cdot)\right)(t)\right) .
$$

Here $P_{U}$ is a projection operator on the control set $U$. For the Projected Gradient Algorithm and for convergence properties of (9) and of some related gradienttype optimization procedures see e.g., $[14,8]$.

Let us now present an implementable computational scheme that follows from our consideration presented above.

Algorithm 1. 1) Choose an admissible initial control $u^{0}(\cdot) \in \mathcal{U}$ and the corresponding continuous trajectory $x^{0}(\cdot)$. Set $k=0$.

2) Given a $x_{q_{i}}^{k}(\cdot)$ define $q_{i+1}^{k}, i=1, \ldots, r+1$ and

$$
t_{i+1}^{k}:=\min \left\{t \in[0,1]: x_{q_{i}}^{k}(t) \bigcap S_{q_{i}, q_{i+1}} \neq \emptyset\right\} .
$$

3) For the determined hybrid trajectory

$$
\mathcal{X}^{u^{k}}=\left(\tau^{k}, x^{k}(\cdot), \bar{q}^{k}\right)
$$

solve the above equations (6) and define the gradient $\nabla \tilde{J}^{*}\left(u^{k}(\cdot)\right)(t) \forall t \in[0,1]$ of the cost functional.

4) Using $\nabla \tilde{J}^{*}\left(u^{k}(\cdot)\right)$, compute the iteration $u^{k+1}(t)$ by using a gradient-type method. Increase $k$ by one and go to Step (2).
Note that the switching times, number of switches and switching sets in the given $O C P(1)$ are assumed to be unknown. Using the iterative structure of the proposed Algorithm 1, one can compute the corresponding approximations of the optimal trajectory, optimal switching times and optimal switching sets. Let us now present the following convergence result for Algorithm 1.

Theorem 8. Assume that the data for the regular $O C P$ (1) satisfies all hypotheses of Section 2 and that (1) has an optimal solution $\left(u^{\text {opt }}(\cdot), x^{o p t}(\cdot)\right)$. Let $\left\{X^{u^{k}}\right\}$ be a sequence of hybrid trajectories generated by Algorithm 1. Then $\left\{X^{u^{k}}\right\}$ is a minimizing sequence for (1), i.e.,

$$
\lim _{k \rightarrow \infty} J\left(\mathcal{X}^{u^{k}}\right)=\phi\left(x^{o p t}(1)\right) .
$$

Finally, note that Theorem 8 can be proved with the help of the dominated convergence theorem and the standard properties of a gradient minimization algorithm in Hilbert spaces.

\section{CONCLUDING REMARKS}

In this paper, we have developed a new approach to a class of hybrid optimal control problems of the Mayer type. This approach is based on explicit formulae for the reduced gradient of the cost functional of the given hybrid optimal control problem. The corresponding relations make it possible to formulate first-order necessary optimality conditions for the considered hybrid optimal control problems and provide a basis for effective computational algorithms. The idea of reduced gradients can also be used for some linearization procedures of the initial optimal control problem. Note that linearization techniques have been recognized for a long time as a powerful tool for solving optimization problems. The approach proposed in this paper can be extended to some other classes of hybrid optimal control problems. Finally, note that it seems to be possible to derive necessary $\epsilon$-optimality conditions (the $\epsilon$-Hybrid Maximum Principle) by means of the presented techniques from Theorem 6 .

\section{REFERENCES}

[1] Azhmyakov, V. (2005). Stable Operators in Analysis and Optimization, Peter Lang, Berlin.

[2] Benedetto, M.D. and A. Sangiovanni-Vincentelli (2001). Hybrid Systems Computation and Control. Springer, Heidelberg.

[3] Berkovitz, L.D. (1974). Optimal Control Theory. Springer, New York.

[4] Branicky, M.S., V.S. Borkar and S.K. Mitter (1998). A unifed framework for hybrid control: model and optimal control theory. IEEE Trans. Automat. Contr. 43, pp. 31-45. 
[5] Caines, P. and M.S. Shaikh (2005). Optimality zone algorithms for hybrid systems computation and control: From exponential to linear complexity. In: Proceedings of the 13th Mediterranean Conference on Control and Automation. pp. 12921297, Limassol.

[6] Cassandras, C., D.L. Pepyne and Y. Wardi (2001). Optimal control of a class of hybrid systems. IEEE Trans. Automat. Contr. 46, pp. 398-415.

[7] Clarke, F.H., Yu.S. Ledyaev, R.J.Stern and P.R. Wolenski (1998). Nonsmooth Analysis and Control Theory, Springer, New York.

[8] Fiacco, A.V. and G. McCormick, (1968). Nonlinear Programming: Sequential Unconstrained Minimization Techniques, Wiley, New York.

[9] Filippov, A.F. (1988). Differential Equations with Discontinuous Right-Hand Sides. Kluwer Academic Publishers, Dordrecht.

[10] Ioffe, A.D. and V.M. Tichomirov (1979). Theory of Extremal Problems. North Holland, Amsterdam.

[11] Morse, A.S., C.C. Pantelides and S. Sastry eds. (1999). Special issue on hybrid systems. Automatica 35.

[12] Piccoli, B. (1998). Hybrid systems and optimal control. In: Proceedings of the 37th IEEE Conference on Decision and Control. pp. 13-18. Tampa.

[13] Piccoli, B. (1999). Necessary conditions for hybrid optimization. In: Proceedings of the 38th IEEE Conference on Decision and Control. pp. 410-415. Phoenix.

[14] Polak, E. (1997). Optimization, Springer, New York.

[15] Pontryagin, L.S., V.G. Boltyanski, R.V. Gamkrelidze and E.F. Mischenko (1962). The mathematical Theory of Optimal Processes. Wiley, New York.

[16] Raisch, J. (1999). Hybride Steuerungssysteme. Shaker, Aachen.

[17] Shaikh, M.S. and P.E. Caines (2004). On the hybrid optimal control problem: The hybrig maximum principle and dynamic programming theorem. IEEE Trans. Automat. Contr. submitted.

[18] Sussmann, H. (1999). A maximum principle for hybrid optimal control problems. In: Proceedings of the 38th IEEE Conference on Decision and Control. pp. 425-430. Phoenix.

[19] Teo, K.L., C.J. Goh and K.H. Wong (1991). A Unifed Computational Approach to Optimal Control Problems, Wiley, New York. 